\title{
An Underwater Thruster Fault Diagnosis Simulator and Thrust Calculation Method Based on Fault Clustering
}

\author{
Jian Yuan $\mathbb{D D}^{1}{ }^{1}$ Junhe Wan, ${ }^{1}$ Wenxia Zhang, ${ }^{2}$ Hailin Liu, ${ }^{1}$ and Hao Zhang $^{1}$ \\ ${ }^{1}$ Institute of Oceanographic Instrumentation, Qilu University of Technology (Shandong Academy of Sciences), \\ Shandong Provincial Key Laboratory of Ocean Environment Monitoring Technology, \\ National Engineering and Technological Research Center of Marine Monitoring Equipment, Qingdao 266100, China \\ ${ }^{2}$ Department of Mechanical and Electrical Engineering, Qindao College, Qingdao 266106, China \\ Correspondence should be addressed to Jian Yuan; yuanjian@qlu.edu.cn
}

Received 18 November 2020; Accepted 16 January 2021; Published 25 January 2021

Academic Editor: Ramadoni Syahputra

Copyright (c) 2021 Jian Yuan et al. This is an open access article distributed under the Creative Commons Attribution License, which permits unrestricted use, distribution, and reproduction in any medium, provided the original work is properly cited.

\begin{abstract}
In order to study the fault diagnosis method of small underwater thruster, an experimental device for fault diagnosis of underwater thruster is designed, and a controller hardware and monitoring software of upper computer and lower computer are developed to realize the acquisition and storage of parameters for underwater propeller. The experimental device can simulate four kinds of thruster faults, collect the hydrophone data, classify the fault types by fault clustering analysis, analyze the spectrum of four types of faults, and calculate the thrust under different fault conditions based on the results of spectrum analysis. The experimental results show that the experimental system effectively simulates different faults of the thruster, and the analysis method realizes the classification of different faults. The thrust loss of different faults is also calculated based on the analysis method.
\end{abstract}

\section{Introduction}

Due to complex marine environment of autonomous underwater vehicle (AUV), the motion states of AUV are interfered by ocean current, waves, and floating objects. The underwater thruster is the most important equipment for AUV's motion control. Once the thruster fails, AUV will not complete the scheduled task and will endanger the safety of the AUV, even leading to AUV loss [1]. Generally, the faults of underwater thruster include motor stalling caused by winding of underwater thruster, propeller output loss caused by propeller missing, and periodic vibration caused by inconsistent installation of propeller shafting, affecting thrust output of thrusters, which will all lead to unstable states of underwater thrusters. For different types of faults, the speed measurement methods of underwater thruster are different. Importantly, there is a lack of effective means of speed measurement. One method is to use a hydrophone to collect acoustic signal of underwater thrusters and then analyze the collected signal according to the blade numbers. The noise and vibration produced by different faults have different characteristics [2]. Therefore, it is necessary to identify different fault types and carry out the corresponding acoustic signal processing method to calculate the propeller speed and then calculate the actual thrust of the thruster. We know that energies in different frequency bands were derived based on Parseval's theorem. Zamanian and Ohadi [3] provided a genetic algorithm and a modified distance function (MDF) to extract optimal feature sets by optimization of the related frequency bands. Yao et al. [4] presented a signal preprocessing method in the original signals of autonomous underwater vehicle, including signal transformation and data fusion based on Dempster-Shafer evidence theory, and then a feature extraction method based on isometric mapping algorithm was proposed to reduce the external disturbance effect and prominently reflect thruster fault. Sun et al. [5] established the six free-space motion equation mathematical models in accordance with the thruster configuration of AUVs. The value of a control loss parameter was adopted on the basis of the models to represent underwater vehicle malfunction, and then a thruster failure model was established. Further, an improved 
Gaussian particle filtering algorithm is proposed and is used to estimate the AUV failure model and motion state. Liu et al. [6] investigated the fault tolerant control for an autonomous underwater vehicle with thruster redundancy, while the AUV is commanded to dock into a station. A Dubins curve is predefined as an optimal docking path for the AUV to follow, and a group of azimuth thrusters are equipped on the AUV to deal with the uncertain faults. Wang et al. [7] utilized a TakagiSugeno fuzzy model to describe the nonlinear plant, and a Markov chain with partly unknown transition probabilities was adopted to characterize the random behaviors of the actuator faults. Combining the utilization of a novel fuzzy singular perturbation-parameter-dependent Markovian Lyapunov function with the introduction of the slack matrix variable, sufficient conditions on the existence of the reliable fuzzy controller were presented. Baldini et al. [8] used dynamic surface control to generate the moment and forces required by the vehicle to perform the desired motion. Then, a fault tolerant thruster allocation policy is employed to distribute the moment and forces among the thrusters. Deep belief network $(\mathrm{DBN})$ is one of the most representative deep learning models. Deng et al. [9] presented an improved quantum-inspired differential evolution to avoid premature convergence and improve the global search ability, and it was further applied to propose a new fault classification. Song et al. [10] proposed a reverse learning mechanism to generate the initial several subpopulations to enhance the convergence velocity and keep the population diversity in subpopulation, which can reduce the computing resources and balance the exploration capability through a cooperative mechanism. In order to improve the search quality, accelerate the convergence, and avoid fall into local optimum and stagnation, Deng et al. [11] developed a novel improved $\mathrm{DE}$ algorithm with the wavelet basis function, which can improve the search quality, accelerate convergence, and avoid fall into local optimum and stagnation. Further, Deng et al. [12] presented a new differential mutation strategy of a difference vector in order to enhance the search and descent ability, and a novel multipopulation mutation evolution method was designed to ensure the relative independence of each subpopulation.

In this paper, an experimental device for fault diagnosis and fault classification method for underwater thruster is provided in order to realize the fault diagnosis of underwater thruster. A set of controller hardware and monitoring software of computer are designed to realize the acquisition and storage of propeller parameters, and the fault simulation and fault classification of four kinds of thrusters are carried out. Furthermore, according to the different types of faults, a kind of calculation method of thrust loss rate and thrust calculation of the underwater thruster is provided. The thrust loss of different faults is also calculated based on the analysis method.

\section{Composition of the Experimental Device}

The fault diagnosis device of underwater thruster includes upper computer, lower computer, power module, electronic governor, propeller, and hydrophone, as shown in Figure 1. The upper computer includes the upper computer control board and data acquisition software. The upper computer control board adopts embedded control board PCM9310, which communicates with the lower computer through one 232 serial port. The propeller data acquisition software completes the acquisition and storage of voltage, current, and speed signals uploaded by the lower computer. The lower computer is located in the control box, and its controller is a microcomputer MEGA128. Its main function is to collect $0-5 \mathrm{~V}$ DC voltage of potentiometer knob through AD (analog to digital conversion) and convert it into a corresponding PWM signal and then output it to an electronic governor through PWM port of the microcomputer, which is used for speed regulation of propulsion motor. The input of the power module is $220 \mathrm{VAC}$, which converts and stabilizes the current to $24 \mathrm{VDC}$ and $5 \mathrm{VDC}$, respectively. The electronic governor is installed in an instrument cabin on the underwater propeller platform. It communicates with the control box through the main cable port, receives the PWM signal from the lower computer, and generates three-phase PWM voltage through the built-in voltage and current regulation program. The brushless motor drives the propeller to adjust the motor speed. The propeller is composed of brushless propulsion motor, coupling, and propeller. The brushless propulsion motor receives the three-phase voltage from the electronic governor and then adjusts the motor speed according to different PWM signals; the coupling connects the motor shaft and the propeller shaft, which can adjust the installation error of shafting of different shafts. In order to simulate the faults of underwater thrusters, different sizes of blades are designed and different propeller defects can be set for each size of propeller. The shafting can be replaced by coupling, and different shafting wear faults can be predefined, and the bearing is designed to be detachable, which can carry out different degrees of bearing failure.

2.1. Upper Computer. The upper computer adopts embedded control board Advantech PCM9310, as shown in Figure 2. It has $12 \mathrm{VDC}$ power supply, $7 \mathrm{~W}$ power consumption, and 4 serial ports. The upper computer control board is installed with Windows 10 system and runs the environment of upper monitoring software .NET framework 4.0 and SQL Server 2017. After opening the power on button on the control panel, the main control panel will be powered automatically. After the system enters the operating system interface, the monitoring software will be automatically loaded, as shown in Figure 3.

2.2. Lower Computer. The lower computer is used to control the forward and reverse rotation and adjust the speed of the propeller and communicate with the upper computer. The control box is equipped with lower computer control board and power supply module. The control box panel is equipped with switch, insurance and speed adjustment knob, main cable interface, serial communication interface, power supply interface, and so on, as shown in Figure 4. The main cable interface is the output of PWM control signal and DC power output of the lower computer, which is connected by high current and three-core aviation plug. The serial 


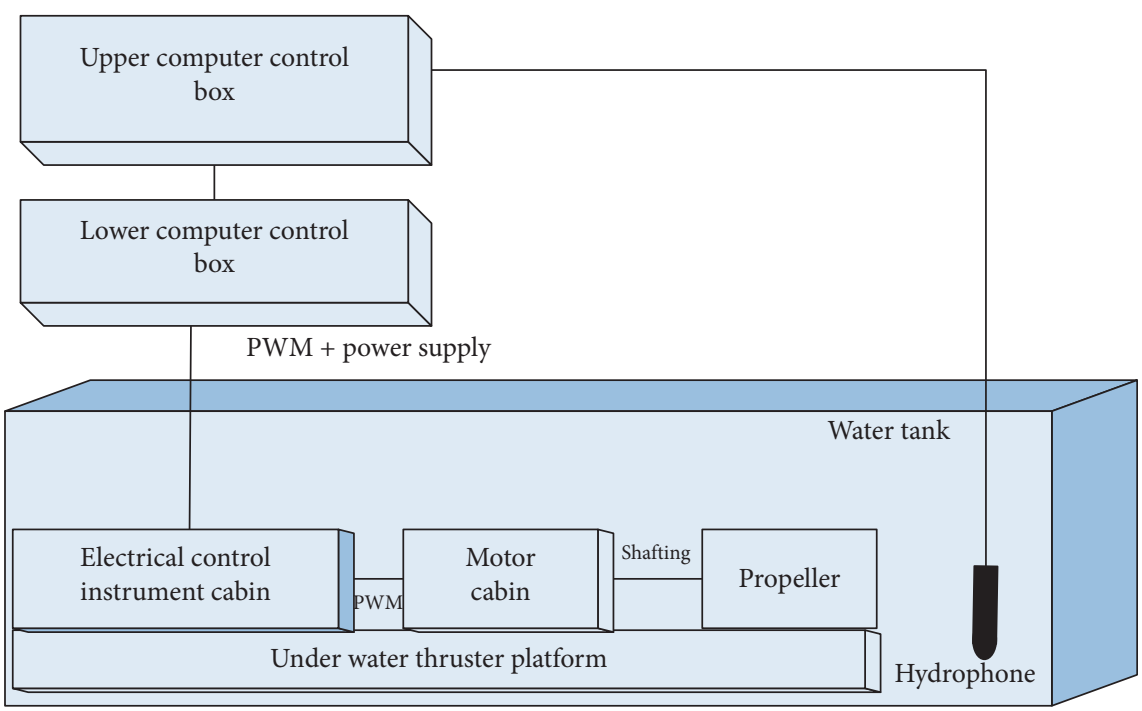

FIgURE 1: Composition of fault diagnosis device for underwater thruster.

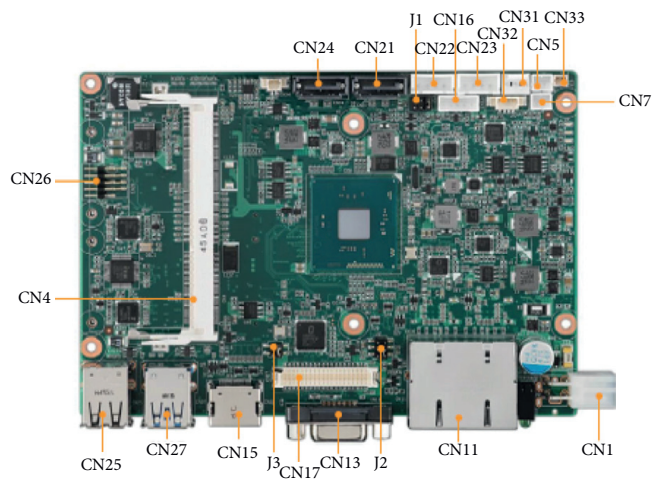

Figure 2: Advantech PCM9310 module for upper computer.

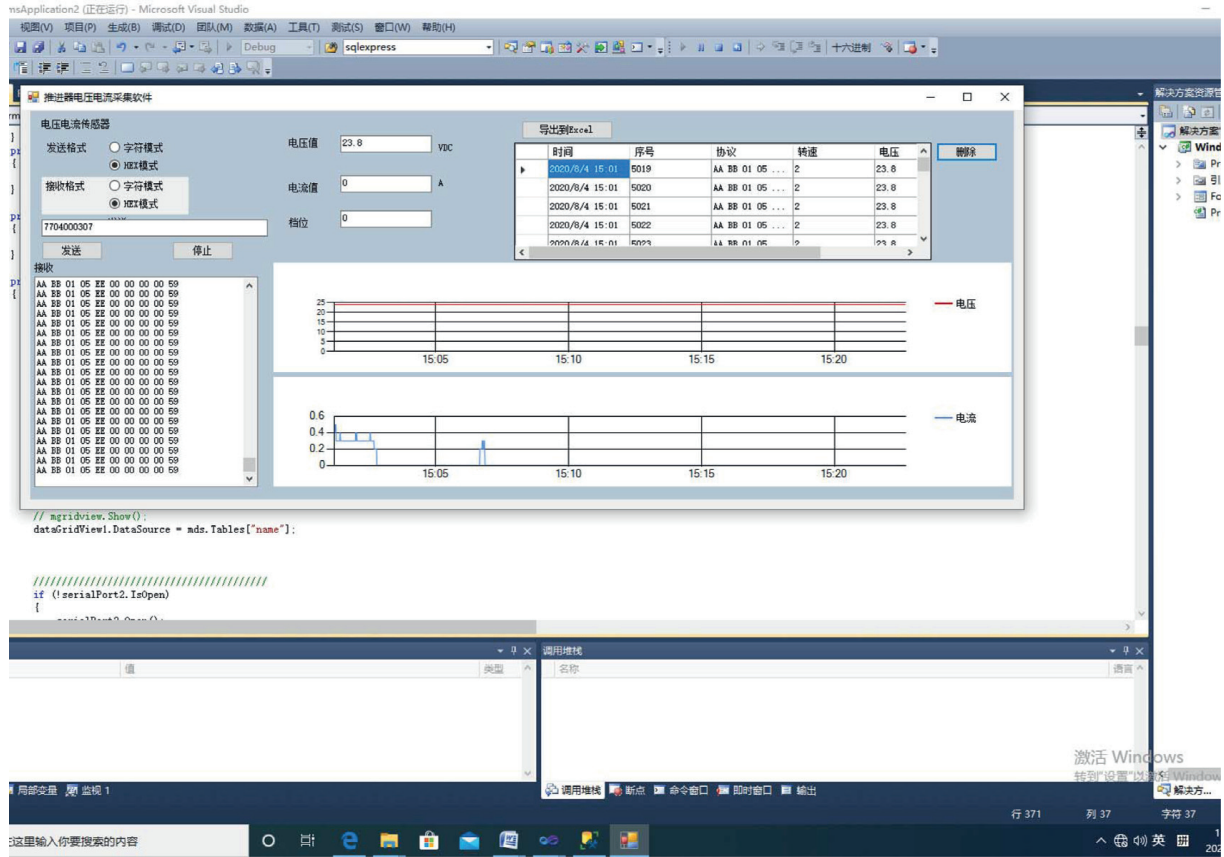

FIgURE 3: Upper computer software and collected data. 


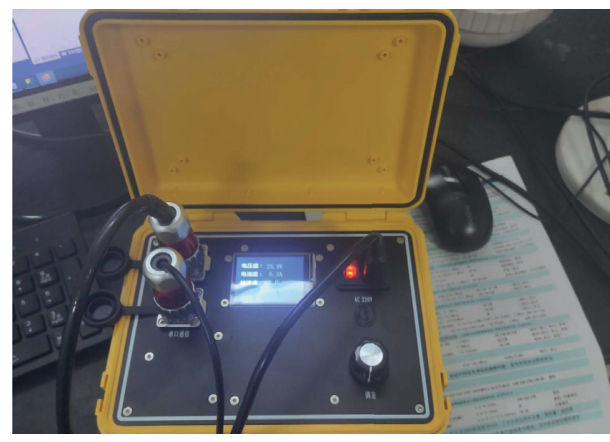

Figure 4: Lower computer control box.

communication interface is connected with the upper computer through TTL-USB module.

The serial port is connected to the "Serial Communication" interface of the panel. The line sequence is defined as follows: yellow: TX, connected to external serial port Rx; black: Rx, not connected; red: GND, connected to external serial port GND. Serial port is TTL level, and the baud rate is 115200 bps.

2.3. Underwater Propeller Platform. The underwater thruster platform includes electronic governor, thruster, and platform. The electronic governor is located in the underwater instrument cabin, which is connected to the main cable output of the control box with the aviation plug. The electronic governor outputs the PWM signal and connects the brushless propulsion motor. The underwater instrument cabin and propeller are fixed on the platform by fixed bearing, as shown in Figure 5.

\section{Software Design}

3.1. Main Functions. The data acquisition software of upper computer is developed based on Visual Studio 2010 framework 4.0 and C\# win Form, shown in Figure 3. The main function is to complete the communication with the lower computer software through the serial port program, so as to realize the real-time acquisition and data storage of the underwater thruster voltage, current, and speed. The data storage software uses SQL Server 2017 database to establish a data table to store the collected voltage, current, speed, and acquisition time. The data acquisition and storage functions are realized by SQL language. The upper computer software can also save the stored data as an Excel file, which is convenient when using MATLAB for postprocessing and fault analysis of the collected data. The lower computer software uses microcomputer AVR mega 128 to collect the analog output of voltage and current detection module and convert it into corresponding voltage and current value according to the numerical conversion function and then generates a real-time data frame.

3.2. Diagnostic Database. According to the requirements of the upper computer software and fault diagnosis function for the voltage, current, speed, and other parameters, a data table is established, as shown in Figure 6. The table

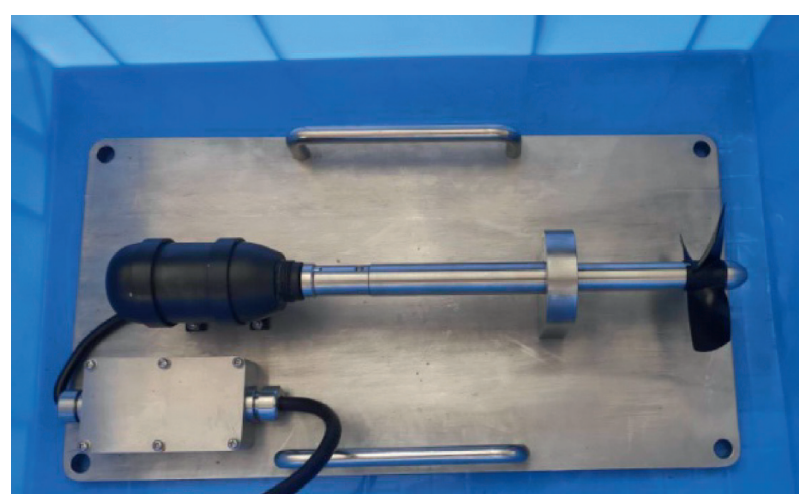

FiguRE 5: Underwater propeller platform.

"Zhenduan" includes no, xieyi, time, vol, cur, speed, and other fields, which is used to store the protocol, storage time, voltage, current, motor speed, and other information collected by the upper computer software, shown in Figure 6 .

3.3. Serial Communication and Protocol Analysis. The serial port communication between the upper computer and lower computer software adopts a sending and receiving protocol to carry out inquiry data communication. The sending and receiving protocol uses hexadecimal format, and the sending intermittent is 1 second. The receiving function of serial port data uses event delegation DataReceived() corresponding to the serial port object to parse the received real-time data. The data frame representation of sending protocol is 77040003 07 and that of receiving protocol is AA BB $0105^{* * * * * * * *}$ (in which "AA BB" denotes the starter of the protocol and "**" denotes the voltage and current data), and the data frame composition is shown in Table 1.

\section{Fault Simulation and Fault Classification}

4.1. Fault Simulation Methods. The device can simulate four kinds of faults.

Fault 1: thrust loss caused by propeller blade failure. According to the fault type, the propeller blade is machined: one of the normal propeller blades is removed, and the propeller state is simulated when one of the propeller blades is missing and the actual speed, theoretical speed, and thrust loss rate of the propeller are monitored and calculated. The actual speed of the propeller is collected by the hydrophone. If one blade of the propeller is missing, the signal generated by the missing blade is equal to a periodic interference signal, and the spectrum intensity is weaker than that of the normal propeller blade.

Fault 2: propeller vibration in shafting. The shafting was processed by EDM tools, and the running states of thruster under the condition of bearing were simulated. The parameters of propeller are collected, and the actual speed, theoretical speed, and thrust loss rate of the propeller are measured and calculated. 


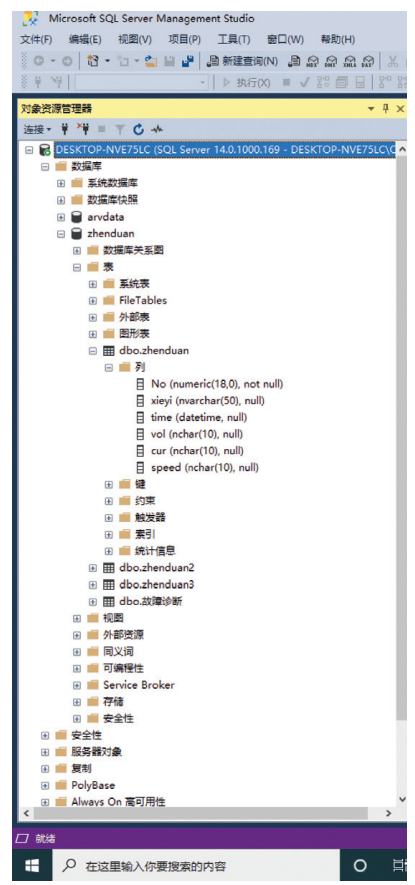

Figure 6: Diagnostic database.

TABle 1: Data frame composition.

\begin{tabular}{|c|c|c|c|c|}
\hline $\begin{array}{l}\text { Frame } \\
\text { header } \\
\text { ( } 2 \text { bytes })\end{array}$ & $\begin{array}{c}\text { Frame } \\
\text { instruction } \\
(1 \text { byte })\end{array}$ & $\begin{array}{c}\text { Frame } \\
\text { length (1 } \\
\text { byte) }\end{array}$ & $\begin{array}{l}\text { Frame } \\
\text { content } \\
\text { ( } 5 \text { bytes })\end{array}$ & $\begin{array}{l}\text { Check sum } \\
\text { (1 byte) }\end{array}$ \\
\hline $\mathrm{AA} B \mathrm{BB}$ & 01 & $\begin{array}{c}\text { Frame } \\
\text { content } \\
\text { length, } 05\end{array}$ & $\begin{array}{c}\text { Low voltage } \\
\text { byte } \\
\text { (note 1) } \\
\text { Voltage } \\
\text { high byte } \\
\text { (note 1) } \\
\text { Current } \\
\text { low byte } \\
\text { (note 2) } \\
\text { Current } \\
\text { high byte } \\
\text { (note 2) } \\
\text { Speed } \\
\text { (note 3) }\end{array}$ & $\begin{array}{l}\text { Frame } \\
\text { header, } \\
\text { frame } \\
\text { instruction, } \\
\text { frame length, } \\
\text { and frame } \\
\text { content }\end{array}$ \\
\hline
\end{tabular}

Voltage value $=($ voltage high byte $<<8+$ voltage low byte $) / 10$; if voltage low byte is $0 \times \mathrm{EE}$, high byte is $0 \mathrm{x} 00$, and voltage value is $23.8 \mathrm{~V}$. Current value $=$ (current high byte $<<8+$ current low byte) $/ 10$; if current low byte is $0 \times 01$, high byte is $0 \times 00$, and current value is $0.1 \mathrm{~A}$. The speed is a signed value, where $0 \times \mathrm{FF}$ indicates reverse 1st gear and $0 \times 01$ indicates 1 st gear; example 1: AA BB $0105 \mathrm{EE} 00010001$ 5B; among them, AA BB is the data header, which does not need to be analyzed; 0105 is the fixed data without parsing; EE 00 is the voltage value; that is, the hexadecimal 00EE is converted to 238 , and the voltage value divided by 10 is 23.8 voltage and low byte is $0 \times \mathrm{EE} \mathrm{V}$.

Fault 3: propeller vibration caused by inconsistent shafting installation. By adjusting the installation height of the coupling and motor cabin, the running state of the propeller under the condition of incon- sistent shafting installation is simulated. The parameters of propeller are collected, and the actual speed, theoretical speed, and thrust loss rate of propeller are measured and calculated.

Fault 4: the motor is blocked by winding. By adjusting the tightening device on the shafting to adjust the speed of the propeller, the running state of the propeller in the case of motor stalling caused by foreign body winding is simulated, and the related parameters of the propeller are collected, and the actual speed, theoretical speed, and thrust loss rate of the propeller are measured and calculated.

\subsection{Fault Classification Calculation Method}

S1. Four types of faults are simulated. After the denoising calculation, the acoustic pressure collected in the aforementioned four types of fault time $T$ is normalized. The data are selected for each type of fault to form a dataset $\Omega_{1 \times p}$, which is a vector of $p$ column. The data in time $T$ is divided into $h$ vectors and the $h$ vectors denotes the different fault types,respectively

The four types of faults include propeller blade missing, propeller vibration in shafting, propeller vibration caused by inconsistent shafting installation, and motor stalling caused by winding.

S2. The dataset in $\mathrm{S} 1$ is initialized by the random number between 0 and 1 , and the membership matrix $U$ is constructed, so that

$$
\begin{aligned}
U= & {\left[u_{11}, u_{12}, \ldots, u_{1 n_{p}}, u_{21}, u_{22}, \ldots,\right.} \\
& \left.u_{2 n_{p}}, \ldots, u_{h 1}, u_{h 2}, \ldots, u_{h n_{p}}\right] .
\end{aligned}
$$

Each data point uses the membership degree to determine the degree belonging to each vector and set $c_{i}$ as the classification center of vector $i$; the membership matrix $U$ satisfies the following constraints:

$$
\sum_{i=1}^{4} u_{i j}=1, \quad j=(1, \ldots, p),
$$

where $u_{i j}$ is the random number between 0 and 1 .

S3. Set the weighted adjustment index and calculate $c_{i}$, and the calculation method is

$$
c_{i}=\frac{\sum_{j=1}^{p} u_{i j}^{m} x_{j}}{\sum_{j=1}^{p} u_{i j}^{m}} .
$$

$m \in(1, \infty)$ is the weighted adjustment index with $m=2$.

S4. Set the optimization function $J(t)$; if $J(t)$ is less than the determined threshold value $J_{1}$, the iteration is stopped. The calculation method of the optimization function $J(t)$ is as follows: 


$$
J(t)=\sum_{i=1}^{4} J_{i}(t)=\sum_{i=1}^{4} \sum_{j}^{p} \frac{u_{i j}^{m} \sum_{j=1}^{p} c_{i} x_{j}}{\sqrt{\sum_{i=1}^{4} c_{i}^{2}} \sqrt{\sum_{j=1}^{p} x_{j}^{2}}}
$$

where $x_{i}$ and $x_{j}$ are the collected data points. If the threshold value $J(t)$ is less than the known determined threshold value, or the change amount $\Delta J(t)$ of $J_{1}$ is less than the determined threshold value $J_{1}$, then the iteration is stopped, where $\Delta J(t)=J(t)-J(t-1)$, the threshold value is 0.001 , and $t$ is the number of iterations.

S5. Update the membership matrix $U$ and return to Step S3, including the following steps.

S51. Calculate $u_{i j}=\sum_{k=1}^{4}\left(d_{i j} / d_{k j}\right)^{(m-1) / 2}$ where

$$
\begin{gathered}
d_{i j}=\frac{\sum_{i=1, j=1}^{p} x_{i} x_{j}}{\sqrt{\sum_{i=1}^{p} x_{i}^{2}} \sqrt{\sum_{j=1}^{p} x_{j}^{2}}}, \\
d_{k j}=\frac{\sum_{i=1, j=1}^{i=4, j=p} c_{i} x_{j}}{\sqrt{\sum_{i=1}^{4} c_{i}^{2}} \sqrt{\sum_{j=1}^{p} x_{j}^{2}}},
\end{gathered}
$$

where $d_{k j}$ denotes the distance between $c_{k}$ and $x_{j} ; d_{i j}$ denotes the distance $x_{i}$ and $x_{j}, k=(1, \ldots, 4)$.

S52. Update the membership matrix $U$ and return to Step S3.

According to the aforementioned fault classification process, the actual thrust of propeller in corresponding fault type can be further calculated. Time complexity of the algorithm is $O\left(I^{*} p^{*} k^{*} m\right)$. Its space complexity is $O\left(p^{*} m\right)$, where $m$ is the number of fields of each element, $n$ is the amount of data, and $I$ is the number of iterations. Generally, $I, k$, and $m$ can be considred as constants, so the time and space complexity can be simplified as $O(p)$ that is linear.

\subsection{Calculation Method of Actual Speed of the Propeller.} The actual speed calculation of thruster considering four faults is as follows:

The actual speed calculation of fault 1: the period of the periodic interference signal $T_{0}$ generated by the missing propeller blade is consistent with the rotation period of the propeller; that is, $T_{1}=T_{0}, f_{1}=\left(1 / T_{1}\right)=f_{0}$, in which $f_{1}$ is the rotation frequency of the propeller and $f_{0}$ is the frequency of the periodic interference signal. By analyzing the frequency spectrum, the main frequency $\omega_{0}$ of the periodic interference signal of the missing screw blade is obtained, and the time-domain frequency of the periodic interference signal of the missing screw blade is calculated according to the frequency calculation formula $f_{0}=\left(\omega_{0} / 2 \pi\right)$, so the speed per minute of the propeller with the missing blade can be obtained with $n_{1}=60 f_{1}=60 f_{0}$.

The actual speed calculation of fault 2: collect the propeller acoustic signal and carry out the frequency domain analysis of the signal to get the main frequency $\omega_{m}$ of the propeller signal. According to the calculation formula $f_{m}=\left(\omega_{m} / 2 \pi\right)$, the time-domain frequency $f_{m}$ is calculated. Because $f_{m}$ is related to the number of blades of the propeller, the rotation speed $n_{2}=60 f_{2}=$ $60\left(f_{m} / m_{p}\right)$ of the propeller, $f_{m}$ is the rotation frequency of the propeller, and $f_{m}=m f_{2}$, in which $f_{2}$ is the rotation frequency of the propeller and $m_{p}$ is the blade number of the propeller.

The actual speed calculation of fault 3: the frequency domain analysis of the signal is carried out, and the frequency spectrum of the signal is obtained. The pe$\operatorname{riod} T_{v}$ of the vibration signal is consistent with the rotation period $T_{3}$ of the propeller; that is, $T_{v}=T_{3}$. The main frequency $\omega_{v}$ of the periodic signal is obtained by analyzing the frequency spectrum, and the time-domain frequency $f_{v}$ of the vibration signal is calculated according to the calculation formula $f_{v}=\left(\omega_{v} / 2 \pi\right)$, so as to obtain the propeller speed with $n_{3}=60 f_{3}=60 f_{v}$.

The actual speed calculation of fault 4 : because of the continuous decrease of propeller speed caused by entanglement, the spectrum of fault signal shows a continuous spectrum of continuous reduction of spectrum intensity. The main frequency $\omega_{e}$ of the signal is obtained through spectrum analysis, and the timedomain frequency $f_{e}$ is calculated according to the frequency calculation formula $f_{e}=\left(\omega_{e} / 2 \pi\right)$, which is related to the number of blades of the propeller, so the speed of the propeller with $n_{4}=60 f_{4}=60\left(f_{e} / m_{p}\right)$ in which $m_{p}$ is the blade number of the propeller.

According to the frequency spectrum of the propeller, the frequency of the propeller can be calculated by calculating the frequency spectrum of the propeller blade.

The thrust $T_{t}$ of underwater thruster is in positive proportion to the square of propeller speed $n, T_{t}=K_{t} \rho n^{2} D^{4}$, where $K_{t}$ is the empirical coefficient of thrust, generally $K_{t}=0.25, \quad \rho$ is the density of water, generally $\rho=10^{3}\left(\mathrm{~kg} / \mathrm{m}^{3}\right), n_{t}$ is the theoretical rotational speed of propeller and its unit is rps $(\mathrm{R} / \mathrm{s})$, and $D$ is the diameter of propeller, and its unit is meter.

From $T_{t}=K_{t} \rho n^{2} D^{4}, K_{t}, \rho$, and $D$ are the known quantities, so $T_{t}=K n^{2}$, and we can get the calculation method of thrust loss rate: thrust loss rate $=$ (theoretical speed $^{2}$-actual speed $\left.^{2}\right) /$ theoretical speed $^{2} \times 100 \%=\left(\left(n_{t}^{2}-\right.\right.$ $\left.\left.n_{i}^{2}\right) / n_{t}^{2}\right) \times 100 \%, \quad i=(1,2,3,4)$.

Further, the calculated actual thrust $=$ theoretical thrust value $\times(1-$ thrust loss rate $)$.

\section{Experimental Analysis}

Using the designed simulator device, we simulate four faults. The hydrophone is used to collect the fault data of 120000 points in a period of time; the processed hydrophone data are shown in Figure 7. Then, we make a further analysis on these data using the proposed fault classification calculation method.

After the signal classification processing, the classification results are shown in Figure 8, and it shows that the data 


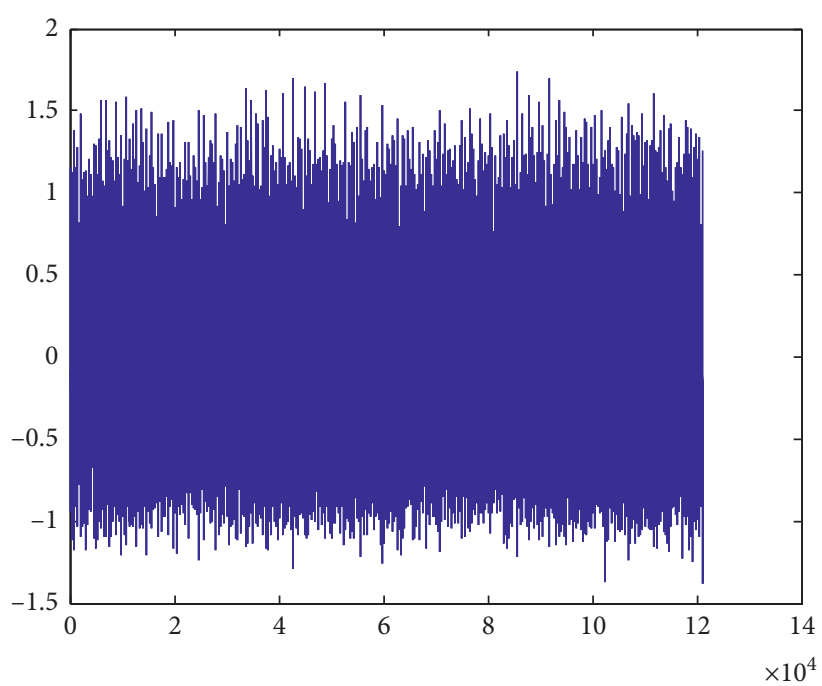

Figure 7: Processed hydrophone data.

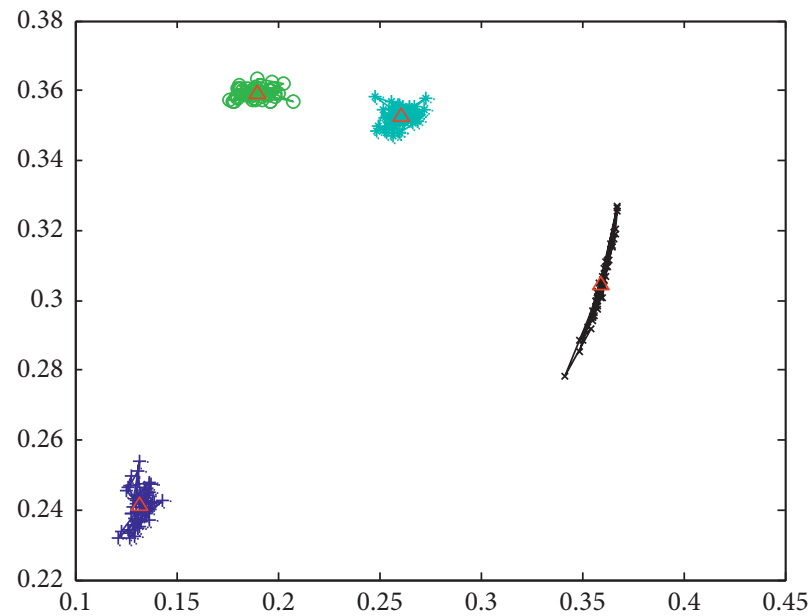

Figure 8: Hydrophone data after fault classification.

points are classified into four types, and each cluster denotes a different fault type. It shows that the algorithm is insensitive to noise and can find clusters of arbitrary shape.

The speed is very fast, because its speed has nothing to do with the number of data objects but only depends on the number of cells in each dimension of the data space. In order to analyze the main frequency of every fault, we make a frequency domain analysis. For each fault, we choose 350 points to analyze with FFT method in order to find the main frequency of each fault, shown in Figures 9-12. For fault 1, the main frequency is about $200 \mathrm{rad}$; for fault 2, the main frequency is about $775 \mathrm{rad}$; for fault 3 , the main frequency is about $207 \mathrm{rad}$; for fault 4, the main frequency is about $700 \mathrm{rad}$.

The propeller has four propeller blades, and the PWM high-level duration is set to $1200 \mu \mathrm{s}$. According to the relationship curve between PWM and speed, the theoretical speed $n_{t}$ is obtained by the relationship curve between the high-level duration of PWM signal and the motor speed. Figure 13 shows the relationship curve between the PWM output high-level duration and the motor theoretical speed.
When the theoretical speed $n_{t}=2000 \mathrm{rpm}=33.3 \mathrm{rps}$ and $D=0.071 \mathrm{~m}$, the theoretical thrust is

$$
T_{t}=K_{t} \rho n_{t}^{2} D^{4}=0.25 \times 10^{3} \times 33.3^{2} \times 0.071^{4}=7(\mathrm{~kg}) .
$$

From $T_{t}=K_{t} \rho n^{2} D^{4}, K_{t}, \rho$, and $D$ are the known, so $T_{t}=K n^{2}$. We can get the thrust loss rate: thrust loss rate $=\left(\right.$ theoretical speed ${ }^{2}-$ actual speed $\left.{ }^{2}\right) /$ theoretical speed $^{2} \times \quad 100 \%=\left(\left(n_{t}^{2}-n_{i}^{2}\right) / n_{t}^{2}\right) \times 100 \%, i=(1,2,3,4)$. Further, the calculated actual thrust $=$ theoretical thrust value $\times(1-$ thrust loss rate). The main frequency $\omega$ of propeller blade rotation signal can be obtained by analyzing the frequency spectrum of dataset $\Omega_{1 \times p}$, in which $p$ is the column of a vector. The time-domain frequency $\omega$ can be calculated according to the frequency calculation formula $f=(\omega / 2 \pi)$. Since $f$ is related to the number of blades of propeller, according to four different fault types and speed formula, the data of propeller can be obtained as shown in Table 2. The theoretical speed in Table 2 is $2000 \mathrm{rpm}$ and the theoretical thrust is $7 \mathrm{~kg}$. 


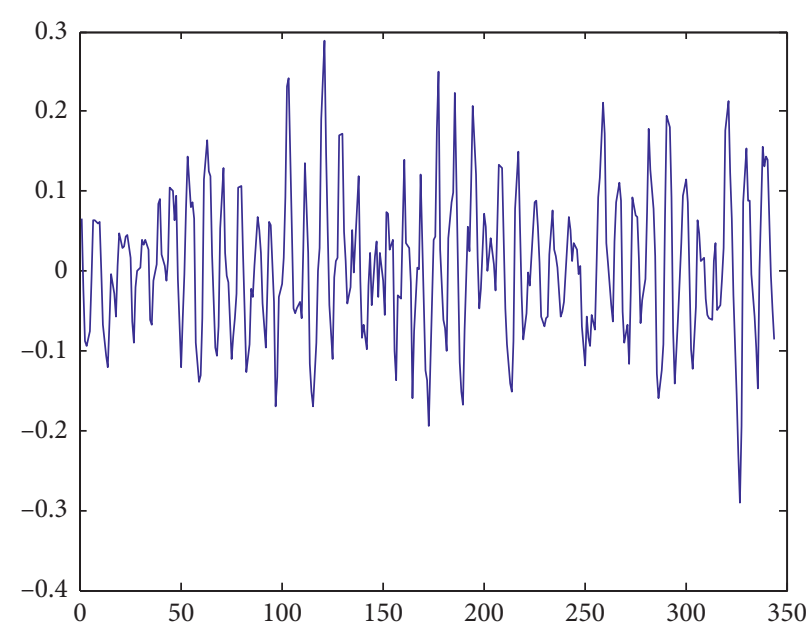

(a)

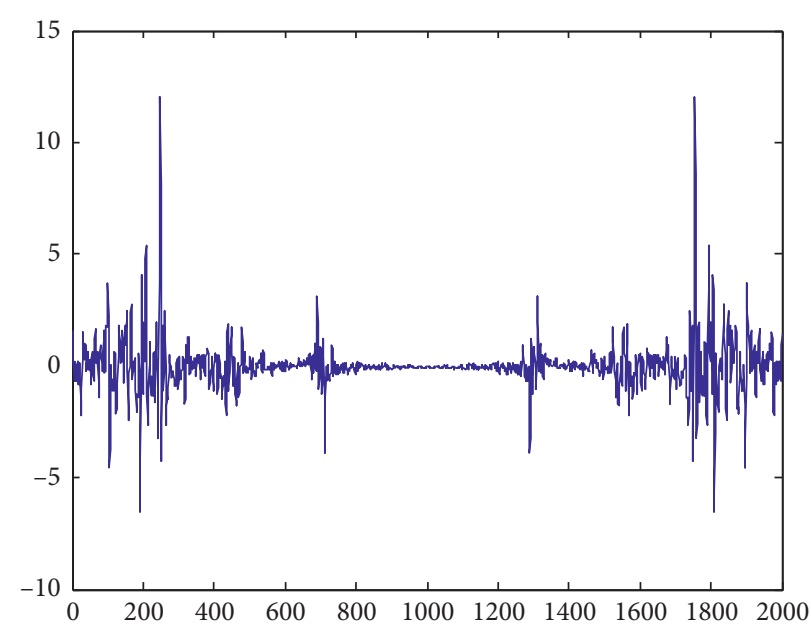

(b)

Figure 9: Partial time-domain data (a) and spectrum analysis (b) of fault 1.

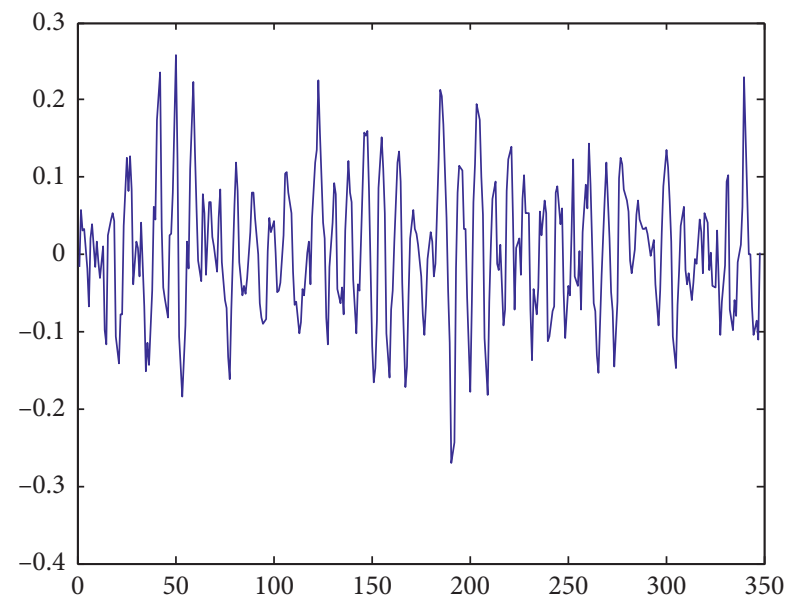

(a)

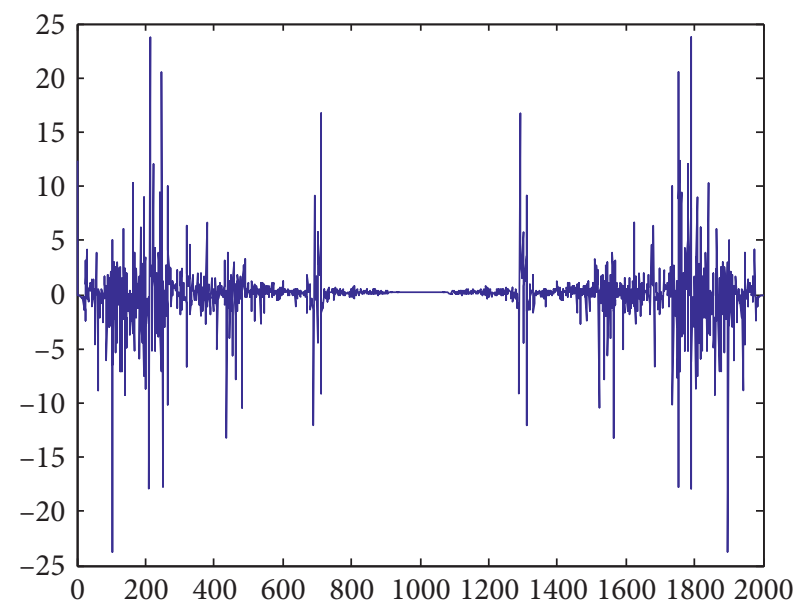

(b)

Figure 10: Partial time-domain data (a) and spectrum analysis (b) of fault 2.

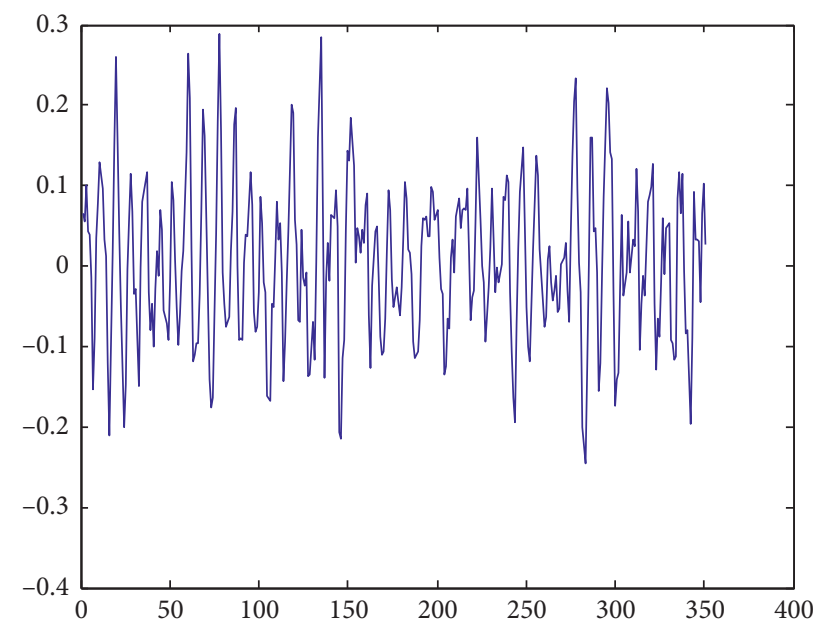

(a)

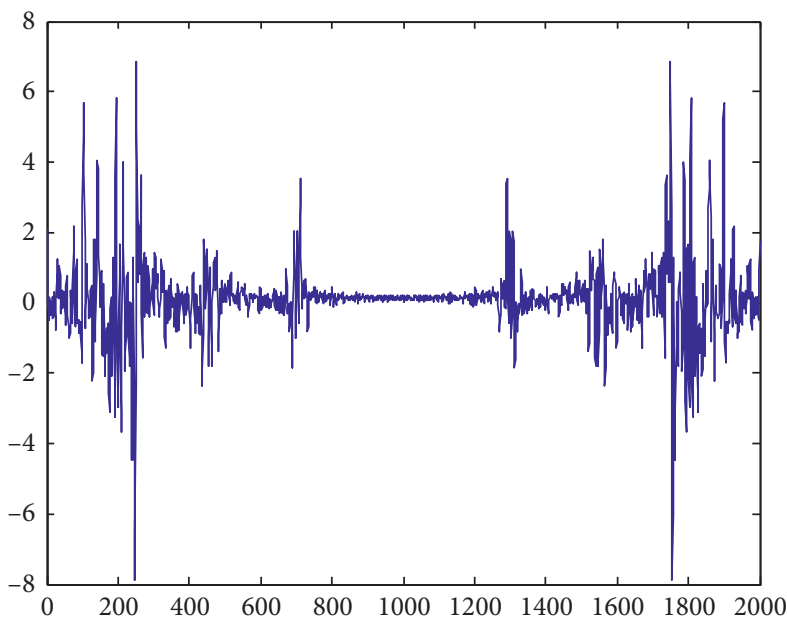

(b)

Figure 11: Partial time-domain data (a) and spectrum analysis (b) of fault 3. 


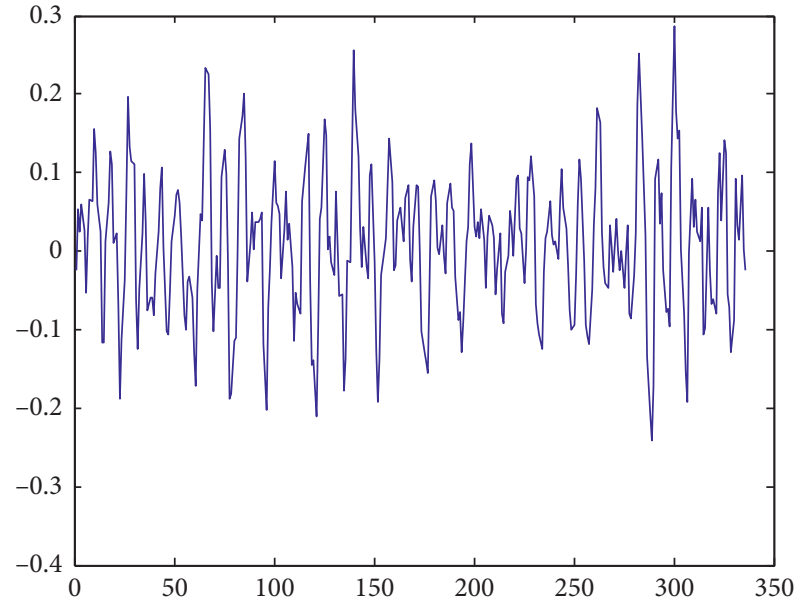

(a)

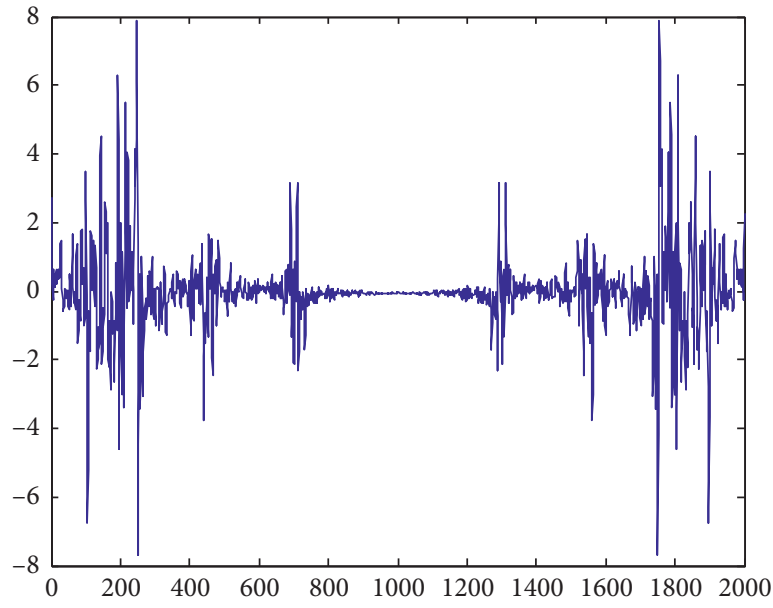

(b)

Figure 12: Partial time-domain data (a) and spectrum analysis (b) of fault 4 .

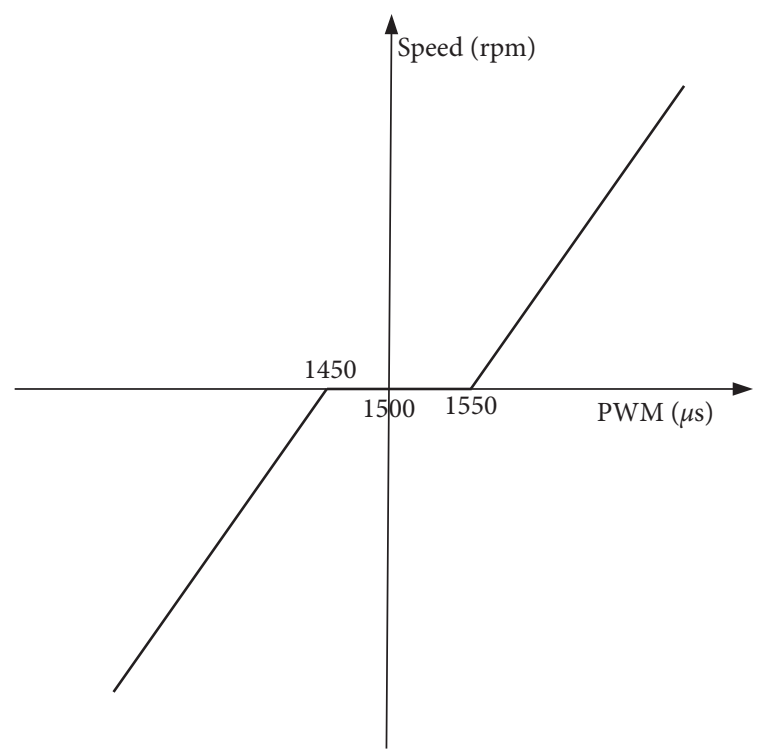

FIGURE 13: Relationship between PWM and motor speed.

TABLE 2: Propeller thrust calculation.

\begin{tabular}{lcccc}
\hline Fault type & $\begin{array}{c}\text { Actual main } \\
\text { frequency } \\
\text { (rad) }\end{array}$ & $\begin{array}{c}\text { Actual } \\
\text { speed (rpm) }\end{array}$ & $\begin{array}{c}\text { Thrust loss } \\
\text { rate (\%) }\end{array}$ & $\begin{array}{c}\text { Measured } \\
\text { thrust (kg) }\end{array}$ \\
\hline Fault 1 & 200 & 1910 & 9 & 6.37 \\
Fault 2 & 775 & 1946 & 5 & 6.65 \\
Fault 3 & 207 & 1977 & 2 & 6.86 \\
Fault 4 & 700 & 1671 & 30 & 4.9 \\
\hline
\end{tabular}

\section{Conclusion}

An experimental device for fault diagnosis and a fault classification method for underwater thruster are provided. A set of controller hardware and monitoring software of computer are designed to realize the acquisition and storage of propeller parameters, and the fault simulation and fault classification of four kinds of thrusters are carried out. Furthermore, according to the different types of faults, a kind of calculation method of thrust loss rate and thrust calculation of the underwater thruster is provided. The thrust loss of different faults is also calculated based on the analysis method.

\section{Data Availability}

The Matlab data files used to support the findings of this study are available from the corresponding author upon request.

\section{Conflicts of Interest}

The authors declare that they have no conflicts of interest.

\section{References}

[1] Y. Chen, Z. Chu, K. Liu et al., "Research progress on thruster fault diagnosis technology for deep-sea underwater vehicle," Journal of Propulsion Technology, vol. 7, 2020.

[2] Y. Fu, Y. Zhang, Y. Gao et al., "Machining vibration states monitoring based on image representation using convolutional neural networks," Engineering Applications of Artificial Intelligence, vol. 65, pp. 240-251, 2017.

[3] A. H. Zamanian and A. Ohadi, "Application of energies of optimal frequency bands for fault diagnosis based on modified distance function," Journal of Mechanical Science and Technology, vol. 31, pp. 2701-2709, 2017.

[4] F. Yao, M. Zhang, and F. Wang, "Weak thruster fault detection for autonomous underwater vehicle based on artificial immune and signal pre-processing," Advances in Mechanical Engineering, vol. 10, no. 2, 2018.

[5] Y. Sun, X.-R. Ran, Y.-M. Li, G.-C. Zhang, and Y.-H. Zhang, "Thruster fault diagnosis method based on Gaussian particle filter for autonomous underwater vehicles," International Journal of Naval Architecture and Ocean Engineering, vol. 8, no. 3, pp. 243-251, 2016.

[6] F. Liu, J. Yu, D. Xu, and L. Bai, "Fault isolation of thrusters under redundancy in frame-structure unmanned underwater 
vehicles," International Journal of Advanced Robotic Systems, vol. 15, no. 2, 2018.

[7] Y. Wang, P. Shi, and H. Yan, "Reliable control of fuzzy singularly perturbed systems and its application to electronic circuits," IEEE Transactions on Circuits and Systems I: Regular Papers, vol. 65, no. 10, pp. 3519-3528, 2018.

[8] A. Baldini, L. Ciabattoni, R. Felicetti, F. Ferracuti, A. Freddi, and A. Monteriù, "Dynamic surface fault tolerant control for underwater remotely operated vehicles," ISA Transactions, vol. 78, 2018.

[9] W. Deng, H. Liu, J. Xu, H. Zhao, and Y. Song, “An improved quantum-inspired differential evolution algorithm for deep belief network," IEEE Transactions on Instrumentation and Measurement, vol. 69, no. 10, pp. 7319-7327, 2020.

[10] Y. Song, D. Wu, Y. Deng et al., "MPPCEDE: multi-population parallel co-evolutionary differential evolution for parameter optimization," Energy Conversion and Management, vol. 228, Article ID 113661, 2021.

[11] W. Deng, J. Xu, Y. Song, and H. Zhao, "Differential evolution algorithm with wavelet basis function and optimal mutation strategy for complex optimization problem," Applied Soft Computing, Article ID 106724, 2020.

[12] W. Deng, J. Xu, X. Gao et al., “An enhanced MSIQDE algorithm with novel multiple strategies for global optimization problems," IEEE Transactions on Systems, Man, and Cybernetics: Systems, 2020. 